\title{
Markers of thrombin and plasmin generation in patients with inherited thrombophilia
}

\author{
L-H Lee, I Jennings, R Luddington, T Baglin
}

\begin{abstract}
Aim-To determine the prevalence of a biochemically detectable hypercoagulable state, defined in terms of increased thrombin or plasmin generation, in patients with phenotypically characterised thrombophilia.

Methods-Plasma concentrations of the prothrombin activation peptide $F 1.2$ and fibrin degradation (FbDP) and fibrinogen degradation products (FgDP) were measured by enzyme immunoassay in 104 patients deficient in natural anticoagulants, and 35 unaffected relatives.

Results-Increased concentrations of F1.2, FbDP, and FgDP were present in 18, 25, and 19 of 104 patients, respectively. There were no correlations between F1.2, FbDP, and FgDP concentrations, or between these parameters and concentrations of natural anticoagulants except for a negative correlation between protein $C$ concentrations and FgDP (rho $=-0.46, p=0.009$ ).

Conclusion-A biochemically detectable hypercoagulable state is present in some patients with asymptomatic thrombophilia. Markers of plasmin generation may be increased more frequently in thrombophilia than markers of thrombin generation. This finding should prompt the inclusion of markers of plasmin generation in prospective longitudinal cohort studies to determine the predictive value of a hypercoagulable state, defined by either excessive thrombin or plasmin generation, for the development of venous thromboembolism.
\end{abstract}

(F Clin Pathol 1994;47:631-634)

Haemostasis and thrombosis depend on the relative production of thrombin and plasmin generated by the coagulation and fibrinolytic systems, respectively. These systems are regulated by natural inhibitors so that a physiological equilibrium exists in which thrombin and plasmin generation are minimal. In thrombophilia an inherited deficiency of antithrombin, protein $C$, or protein $S$ may predispose some individuals to venous thromboembolism. While there is a recognised association between thrombophilia and overt thrombosis in some pedigrees, the risk of thrombosis is variable within and between families. An attempt has therefore been made to define a hypercoagulable state characterised by exces- sive thrombin generation. The correlation between this biochemically defined hypercoagulable state and the subsequent development of clinical thrombosis might then be determined from prospective longitudinal cohort studies. The ability to identify individuals with thrombophilia who are at high risk of venous thrombosis would mean that antithrombotic prophylaxis could be offered to them. The ability to quantify the hypercoagulable state might also allow the antithrombotic efficacy of anticoagulant treatment to be monitored, which would in turn lead to the development of effective low intensity anticoagulant regimens.

The demonstration of concomitant plasmin and thrombin generation in patients with thrombosis is important and may be relevant to the definition of a hypercoagulable state in thrombophilia (Richards $\mathrm{M}$, et al. Abstract presented at the Congress of the International Society of Haematology Proceedings, London, 1992). In cross-sectional studies increased concentrations of markers of thrombin generation are found in $25 \%$ of patients with inherited thrombophilia. ${ }^{1-3}$ Assessment of thrombophilia syndromes with markers of plasmin generation may complement measurement of thrombin generation, and the relative predictive values of markers of plas$\mathrm{min}$, as well as thrombin generation for the subsequent development of thrombosis, require clarification. We therefore performed a cross-sectional study to confirm the prevalence of a hypercoagulable state, defined by excessive thrombin generation, in patients with a variety of inherited thrombophilic syndromes.

\section{Methods}

Plasma concentrations of antithrombin, protein $\mathrm{C}$, and protein $\mathrm{S}$ were determined in 80 healthy blood donors (46 men, 34 women, mean age 36 years). Antithrombin concentrations were normally distributed and deficiency was defined as a level less than 2 standard deviations below the mean $(<78 \%)$. Protein C concentrations were log normally distributed and deficiency was defined as a concentration less than 2 standard deviations below the mean of the log transformed data $(<73 \%)$. Free protein $S$ concentrations were $\log$ normally distributed and were significantly different in men and women. Protein S deficiency was defined as a concentration less than 2 standard deviations below the mean (men $<71 \%$ and women $<54 \%$ ). 
Characteristics of thrombophilic and control groups

\begin{tabular}{llllll}
\hline Patient group & Number & Pedigrees & $\begin{array}{l}\text { Age: } \\
\text { median and range }\end{array}$ & Malelfemale & $\begin{array}{l}\text { Thrombotic history } \\
\text { yes/no }\end{array}$ \\
\hline All thrombophilia patients & 106 & 87 & 37 & $45 / 59$ & $81 / 25$ \\
Antithrombin deficiency & 18 & 18 & $34(24-61)$ & $7 / 11$ & $18 / 0$ \\
Protein C deficiency & 34 & 27 & $37(11-70)$ & $15 / 19$ & $26 / 8$ \\
Protein S deficiency & 54 & 42 & $37(8-75)$ & $23 / 29$ & $37 / 17$ \\
Controls & 35 & 12 & $35(5-62)$ & $13 / 22$ & $0 / 35$ \\
\hline
\end{tabular}

Between July 1988 and March 1993 venous blood samples anticoagulated in 0.109 molar sodium citrate were collected from patients with a personal or family history of venous thromboembolism before the age of 45 . Plasma was stored in aliquots at $-80^{\circ} \mathrm{C}$ until analysis. A total of 104 patients (45 men and 59 women) were identified with thrombophilia who were asymptomatic and untreated at the time of blood sampling. Eighty one had a history of deep vein thrombosis or pulmonary embolism and 25 were affected family members with no history of thrombosis. Samples from 35 unaffected family members were used as controls.

Antithrombin III and protein $\mathrm{C}$ were measured by chromogenic assay on an ACL coagulometer (Instrumentation Laboratory UK Ltd, Warrington, Cheshire). Antithrombin III was measured using the commercial Immuno kit (Immuno Ltd, Sevenoaks, Kent). Protein $\mathrm{C}$ was measured using Kabi Coamate Protein C kit (Kabi Quadratech, Epsom, Surrey).

Free protein $S$ was measured by enzyme linked immunosorbent assay (ELISA) in plasma supernatant fluids following precipitation with $20 \%$ polyethylene glycol. ${ }^{4}$ Primary and secondary peroxidase conjugated antibody were obtained from Dako (High Wycombe, Bucks).

F1.2 concentrations were measured in a commercial two stage ELISA-Thrombonostica F1.2 (Organon Technika, Cambridge).
The primary antibody was a monoclonal F1.2 specific murine antibody and the secondary conjugate antibody was a rabbit antibody labelled with horseradish peroxidase.

Fibrinogen degradation products (FgDP) and fibrin degradation products (FbDP) were measured using commercial ELISAsFibrinostika FbDP and Fibrinostika FgDP (Organon-Technika).

For each assay the number of patients with thrombophilia who had plasma concentrations of markers above the normal limit was determined. The normal limit was set at the 95th percentile of the distribution of markers in the unaffected family members. Correlations between different markers and between individual markers and concentrations of natural anticoagulants were determined using the Spearman rank correlation coefficient.

\section{Results}

Samples obtained from the 104 patients fulfilled predetermined diagnostic criteria for thrombophilia. Patients were asymptomatic with no treatment at the time of blood sampling. Thirty five relatives of these patients who were asymptomatic and did not have thrombophilia were used as the control group to determine the normal ranges for markers in healthy age and sex matched individuals living in the same environment as the patient

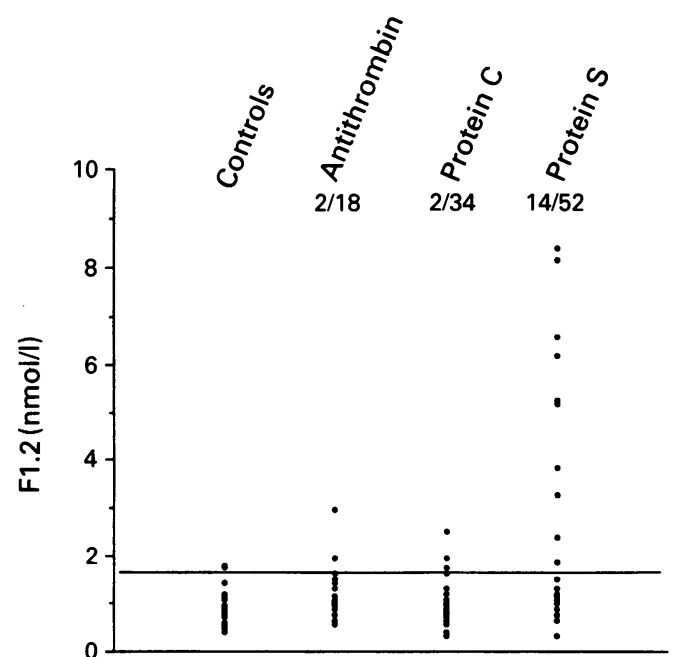

Figure 1 F1.2 concentrations in controls and patients with thrombophilia. The horizontal line shows the upper limit of normal and the numbers of patients with raised values are indicated.
Figure 2 FbDP concentrations in controls and patients with thrombophilia. The horizontal line shows the upper limit of normal and the numbers of patients with raised values are indicated. 
Figure 3 FgDP concentrations in controls and patients with thrombophilia. The horizontal line shows the upper limit of normal and the numbers of patients with raised values are indicated.

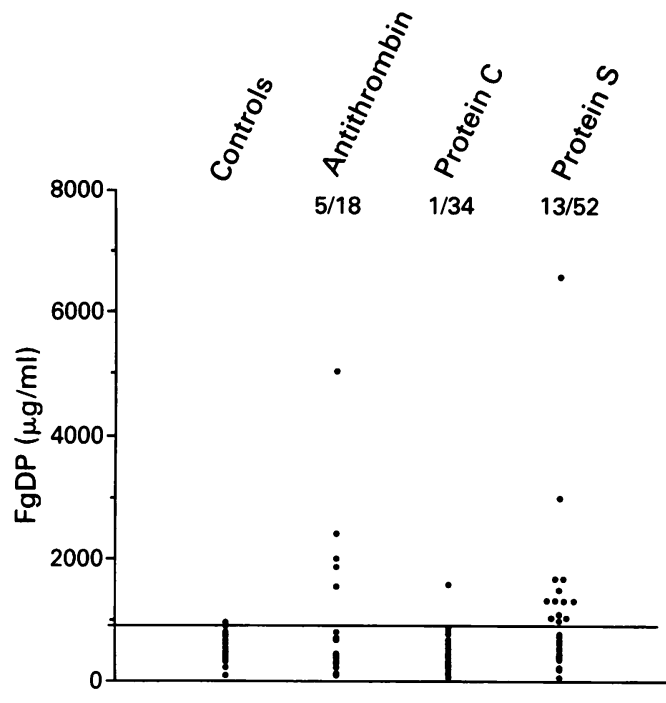

groups. The table shows the characteristics of the thrombophilic and control groups.

The 95th percentiles for the non-affected family members were, respectively, F1.2 1.74 $\mathrm{nmol} / \mathrm{l}$, FbDP $779 \mu \mathrm{g} / \mathrm{ml}$, and FgDP 904 $\mu \mathrm{g} / \mathrm{ml}$. Figures 1-3 show the individual results, with the upper limit of normal in the control group indicated by the horizontal solid lines. The number of patients with concentrations above the upper limit are also indicated on the graph. Although all four markers seemed to be higher in patients with a history of thrombosis, the differences did not reach significance (Student's $t$ test).

There was no correlation between F1.2, $\mathrm{FbDP}$, or FgDP concentrations in any of the thrombophilic groups (antithrombin, protein C, protein S). There was no correlation between concentrations of natural anticoagulants and markers of thrombin or plasmin generation in the antithrombin or protein $S$ deficient patients. However, in patients with protein $\mathrm{C}$ deficiency there was a significant negative correlation between protein $\mathrm{C}$ and FgDP concentrations (rho $=-0.46, \quad \mathrm{p}=$ $0 \cdot 009$ ). There was no correlation between protein $\mathrm{C}$ and $\mathrm{F} 1.2$, or FbDP concentrations.

\section{Discussion}

The aims of this study were to determine the prevalence of a biochemically detectable hypercoagulable state, defined in terms of increased thrombin or plasmin generation in patients with phenotypically characterised thrombophilia. An initial report of raised F1.2 concentrations in 11 of $22(50 \%)$ patients with antithrombin deficiency, compared with their unaffected siblings, ${ }^{5}$ was subsequently explained by ex vivo thrombin generation when blood samples were anticoagulated with heparin. ${ }^{6}$ Subsequent studies incorporating citrate as anticoagulant and ELISA methodology for F1.2 measurement found increased concentrations in only four of $26(15 \%)$ patients from 16 kindreds with phenotypically characterised antithrombin deficiency. ${ }^{7} \mathrm{~A}$ further study in 22 affected members of a single kindred with a type 2 antithrombin variant (antithrombin Hamilton) found increased levels of F1.2 in only five (23\%). Similarly, Mannucci's study found only 14 of 57 (25\%) patients with protein $\mathrm{C}$ deficiency and seven of $24(29 \%)$ with protein S deficiency with raised $\mathrm{F} 1.2$ concentrations. ${ }^{7}$ Thus in crosssectional studies few patients with thrombophilia have a detectable hypercoagulable state, as defined by excessive activation of the common pathway and cleavage of the F1.2 fragment from prothrombin to yield thrombin. Whether these patients are at highest risk for the development of thromboembolism will only be determined by prospective longitudinal cohort studies.

In patients with established venous thromboembolism markers of plasmin generation have generally been found to be increased more frequently than markers of thrombin generation, though the rapid reduction in the latter, following the start of anticoagulant treatment, has been a consideration. ${ }^{8}$ However, in a recent direct comparative study of markers of activation of the haemostatic system in untreated patients with venous thromboembolism, we were able to confirm that markers of plasmin generation are increased more frequently than markers of thrombin generation (Richards $\mathrm{M}$, et al. Abstract presented at the Congress of the International Society of Haematology Proceedings, London, 1992). The demonstration of concomitant plasmin and thrombin generation in patients with thrombosis is important and may be relevant to the definition of a hypercoagulable state in thrombophilia, as the detection of a hypercoagulable state defined by increased concentrations of markers of plasmin generation may complement those of thrombin generation.

In this study we confirmed that increased F1.2 concentrations are detectable in only $17 \%$ of patients with a variety of phenotypically characterised inherited thrombophilia syndromes. Interestingly F1.2 concentrations are more frequently raised in protein $\mathrm{S}$ deficiency than in other thrombophilic syndromes, a finding also observed by others. ${ }^{7}$ This requires explanation. We have also shown that markers of plasmin generation are more frequently raised in thrombophilia than markers of thrombin generation $(24 \%$ of all patients, $28 \%$ with antithrombin deficiency, $26 \%$ with protein $C$ deficiency, $21 \%$ with protein $\mathrm{S}$ deficiency). This finding should prompt the inclusion of markers of plasmin generation in prospective longitudinal cohort studies to determine the predictive value of a hypercoagulable state, defined by either excessive thrombin or plasmin generation, for the development of venous thromboembolism. A direct comparative evaluation of markers of thrombin and plasmin generation in other prethrombotic conditions, such as the antiphospholipid syndrome, ${ }^{9}$ is also warranted.

This work was supported by a grant from the Cambridge Hospitals Trust Funds. L-H Lee is a visiting Research Fellow supported by a scholarship from The Health Manpower Development Plan, Singapore. 
1 Bauer K, Rosenberg R. The pathophysiology of the prethrombotic state in humans: insights gained from prethrombotic state in humans: insights gained from Blood 1987;70:343-50.

2 Mannucci P, Giangrande P. Detection of the prethrombotic state due to procoagulant imbalance. Eur $f$ Haematol 1992;48:65-9.

3 Demers C, Ginsberg J, Henderson P, Ofosu F, Weitz J Blajchman M. Measurement of markers of activated coagulation in antithrombin III deficient subjects. Thromb Haemostas 1992;67:542-4.

4 Woodham $B$. The simultaneous measurement of total and free protein S by ELISA. Thromb Res 1988;50:213-30.

5 Bauer K, Goodman T, Kass B, Rosenberg R. Elevated factor Xa activity in the blood of asymptomatic patients with congenital antithrombin deficiency. $f$ Clin Invest 1985;76:826-36.
6 Bauer K, Barzegar S, Rosenberg R. Influence of anticoagulants used for blood collection on plasma procoagulants used for blood collection on plasma pro-
thrombin fragment $\mathrm{F} 1+2$ measurements. Thromb Res 1991;63:617-28.

7 Mannucci P, Tripodi A, Bottasso B, et al. Markers of procoagulant imbalance in patients with inherited thrombophilic syndromes. Thromb Haemostas 1992;67:200-2.

8 Boneu B, Bes G, Pelzer H, Sie P, Boccalon H. D-dimers, thrombin antithrombin III complexes and prothrombin fragments $1+2$ : Diagnostic value in clinically suspected deep vein thrombosis. Thromb Haemostas 1991;65: 28-32.

9 Ginsberg J, Demers C, Brill-Edwards P, et al. Increased thrombin generation and activity in patients with systemic lupus erythematosus and anticardiolipin antibodtemic lupus erythematosus and anticardiolipin antibod-
ies: evidence for a prothrombotic state. Blood 1993;81: $2958-63$. 